\title{
Evidências de Validade de Marcadores Reduzidos para a Avaliação da Personalidade no Modelo dos Cinco Grandes Fatores ${ }^{1}$
}

\author{
Nelson Hauck Filho \\ Wagner de Lara Machado \\ Marco Antônio Pereira Teixeira \\ Denise Ruschel Bandeira \\ Universidade Federal do Rio Grande do Sul
}

\begin{abstract}
RESUMO - Instrumentos psicométricos breves para a avaliação da personalidade têm recebido uma crescente atenção. Até o momento, entretanto, existia a carência de tais instrumentos no contexto brasileiro. O objetivo do presente estudo foi avaliar as propriedades psicométricas de um instrumento reduzido para mensurar os Cinco Grandes Fatores (CGF), derivado de um instrumento originalmente com 64 marcadores. Os participantes foram 674 estudantes universitários (média de idade $=23,5$; $D P=6,46)$. Foram utilizados critérios teórico-semânticos e estatísticos para eleger um conjunto reduzido de marcadores. A solução fatorial final contou com 25 marcadores, sendo cinco para cada um dos CGF, explicando 53,92\% da variância. Ao final, discute-se a necessidade de estudos subsequentes avaliando a validade convergente do instrumento, bem como potencialidades do mesmo.
\end{abstract}

Palavras-chave: personalidade; Cinco Grandes Fatores; instrumento de avaliação; análise fatorial

\section{Validity Evidences of Mini-markers for Assessing the Big Five Personality Model}

\begin{abstract}
Brief psychometric measures for personality assessment have received increasing attention in the psychological literature. Up to date, however, there was a lack of brief personality measures in Brazil. The present study explored the psychometric properties of Big Five mini-markers derived from an instrument originally composed of 64 items. Participants were 674 undergraduate students (mean age $=23.5$ years; $S D=6.46$ ). Exploratory factor analyses as well as theoretical-semantic criteria were used to select a brief set of personality markers. The final factor solution was composed of 25 markers, five for each Big Five dimension, explaining 53.9\% of the variance. Finally, we discuss the need to investigate the convergent validity of the measure, and considerations regarding its potential usefulness.
\end{abstract}

Keywords: personality; Five-Factor Model; psychometric instrument; factor analysis

A hipótese léxica é um postulado teórico segundo o qual todas as culturas codificam, na linguagem, fenômenos sociais e naturais relevantes, tais como aspectos salientes da idiossincrasia dos indivíduos (John, Angleitner, \& Ostendorf, 1988; Revelle, 1995). Assim sendo, uma das implicações da hipótese léxica é que os termos linguísticos utilizados pelos indivíduos leigos para descrever características individuais (e.g., adjetivos) são relevantes para o estudo dos agrupamentos semânticos representativos dos traços básicos da personalidade humana (Goldberg, 1993). Em virtude disso, ao final do século XIX, surgiu uma série de investigações sobre os descritores linguísticos da personalidade usualmente empregados em diversas culturas (Digman, 1990; Goldberg, 1993; e John, Angleitner, \& Ostendorf, 1988). Essa linha de pesquisa, que foi potencializada pela difusão de técnicas

1 Os autores gostariam de prestar seus sinceros agradecimentos aos dois pareceristas anônimos e ao Editor Associado Fabio Iglesias pelas suas valiosas sugestões que ajudaram a aprimorar a versão anterior deste manuscrito.

2 Endereço para correspondência: Universidade Federal do Rio Grande do Sul - Instituto de Psicologia. Rua Ramiro Barcelos, 2600, sala 117. Bairro Santana, CEP: 90035-003 - Porto Alegre, RS - Brasil. Telefone: (51) 33165454.E-mail: hauck.nf@gmail.com. estatísticas como a análise fatorial, deu início à busca por uma taxonomia unificada dos traços de personalidade.

Um dos primeiros autores a demonstrar interesse pela análise do léxico no estudo dos traços da personalidade foi Francis Galton, no século XIX, que listou mais de 1000 termos em inglês popularmente usados para descrever traços de personalidade (John, Angleitner, \& Ostendorf, 1988). O trabalho de Galton, por sua vez, inspirou as primeiras análises sistemáticas de termos linguísticos na década de 20 do século passado, realizadas pelos autores Franziska Baumgarten em 1926, a partir do idioma alemão, e por Gordon Allport e Henry Odbert em 1936, a partir do inglês (Goldberg, 1993). Subsequentemente, Raymond Cattell, em 1946, aprofundou a investigação dos descritores da personalidade em inglês, apresentando 67 agrupamentos semânticos a partir de análises léxicas e derivações de teorias personológicas da época (John, Angleitner, \& Ostendorf, 1988). No entanto, em 1949, Donald Fiske, com o objetivo de reduzir a personalidade a um conjunto parcimonioso de aspectos fundamentais, constatou, mediante análises fatoriais, serem necessárias apenas cinco variáveis para representar os dados originais de Cattell (Goldberg, 1993). Após esse trabalho, diversos autores (e.g., E. C. Tuppes, R. E. Christal e W. Norman), entre as décadas de 50 e 60 do século passado, relataram 
cinco fatores básicos análogos aos de Fiske em seus estudos (Costa \& McCrae, 1992; Goldberg, 1993; John, Angleitner, \& Ostendorf, 1988). Esses fatores, atualmente replicados em diversas culturas (Hendriks \& et al., 2003), em diferentes etapas do desenvolvimento humano (Kandler \& et al., 2010) e mesmo em outras espécies de mamíferos (Bouchard \& Loehlin, 2001), têm sido chamados de os Cinco Grandes Fatores da personalidade (CGF).

O modelo dos CGF é uma organização hierárquica da personalidade em termos de cinco dimensões fundamentais: extroversão, socialização, conscienciosidade, neuroticismo e abertura. A posição de um indivíduo no continuum extroversão indica o quanto ele é assertivo, ativo socialmente, comunicativo e reforçado por emoções positivas, enquanto a posição no continuum socialização caracteriza o quanto apresenta tendências pró-sociais, como lealdade, generosidade, flexibilidade, altruísmo e modéstia (Costa \& McCrae, 2007). A dimensão conscienciosidade envolve características como competência, determinação, organização, disciplina e persistência, enquanto neuroticismo abrange ansiedade, raiva, depressão, impulsividade e afetos negativos em geral (Costa \& McCrae, 2007). Abertura, por sua vez, abarca aspectos como a curiosidade intelectual, a flexibilidade quanto às crenças pessoais e o gosto pela complexidade, pela variedade e por experiências novas e não-convencionais (Vasconcellos \& Hutz, 2008).

\section{As perspectivas ortogonal e oblíqua na concepção dos CGF}

Os CGF são considerados, em uma perspectiva teórica ortogonal, como fatores independentes entre si e que esgotam a ordem superior da hierarquia dos traços da personalidade. Nessa perspectiva, são propostos fatores inferiores (facetas), representativos das múltiplas possibilidades semânticas do continuum de cada um dos CGF. Assim, por exemplo, o fator extroversão é uma dimensão ampla, representável por facetas tais como nível de comunicação, altivez, assertividade e interações sociais, enquanto a socialização é representável pelas facetas amabilidade, pró-sociabilidade e confiança nas pessoas (Nunes, 2007). Com algumas exceções, os estudos psicométricos pioneiros sobre os CGF inseriam-se dentro dessa perspectiva, como evidenciado pela popularização dos métodos de rotação ortogonais de análise fatorial (e.g., varimax) nessa área de pesquisa (Ruiz \& Jiménez, 2004).

Em contraste, uma perspectiva oblíqua entende os CGF como inter-relacionados (correlacionados). Essas correlações, por sua vez, para alguns autores, são indicativas da existência de fatores superiores aos CGF na hierarquia dos traços, como os fatores Alpha e Beta e o Fator Geral da Personalidade (FGP) (Digman, 1997; Mount, Barrick, Sculen, \& Rounds, 2005; Musek, 2007; Rushton \& Irwing, 2008; van der Linden, te Nijenhuis, \& Bakker, 2010; Zawadzki \& Strelau, 2010). O fator superior Alpha é assumido como responsável pela relação entre as dimensões socialização, conscienciosidade e neuroticismo, sendo também denominado estabilidade. Uma possível interpretação para esse fator é que representa um conjunto amplo de traços rela- cionados à adaptação ao ambiente social. Enquanto isso, o fator Beta explica a extroversão e a abertura, sendo também conhecido como plasticidade, sendo sua interpretação feita em termos de aspectos da aprendizagem e da exploração do ambiente social.

O FGP, por sua vez, pode ser concebido tanto explicando diretamente correlações entre os CGF (Musek, 2007) quanto explicando possíveis correlações entre os fatores superiores Alpha e Beta (Rushton \& Irwing, 2008). O significado teórico atribuído ao FGP é descrito como uma mistura de traços de estabilidade e plasticidade, representativos de adaptações psicológicas evolutivas à vida em sociedade (Just, 2011). Contudo, apesar de um FGP ser uma explicação plausível do ponto de vista estatístico para o padrão recorrente de correlações entre os CGF, existe ainda uma discussão sobre o status ontológico do FGP (bem como dos fatores hierárquicos Alpha e Beta). Ou seja, ainda não há um consenso sobre se são dimensões psicológicas genuínas ou apenas artefatos metodológicos. Em um estudo, por exemplo, os autores relataram que modificar os itens de um instrumento de modo a reduzir a influência da desejabilidade social nas sentenças pode levar a uma redução substancial nas correlações entre os CGF (Bäckström, Bjorklund, \& Larsson, 2009).

\section{O estudo e a avaliação dos CGF no Brasil}

A rede nomológica dos CGF inclui um vasto rol de variáveis, pertencentes tanto ao domínio das diferenças individuais (e.g., psicopatologia e saúde psicológica), quanto dos aspectos interpessoais (e.g., relacionamentos entre pares e relacionamentos familiares) e mesmo dos aspectos sociais e institucionais (e.g., atitudes e valores) (Ozer \& Benet-Martínez, 2006). Dessa forma, no Brasil, o modelo dos CGF tem sido utilizado para aprofundar o conhecimento em diversas áreas temáticas dentro da Psicologia. Exemplos são estudos sobre o bem-estar subjetivo (Nunes, Hutz, \& Giacomoni, 2009), sobre as habilidades sociais (Bartholomeu, Nunes, \& Machado, 2008) e sobre a agressividade (Sisto \& Oliveira, 2007). Silva e Nakano (2011) realizaram um levantamento sobre a produção científica nacional relacionada aos CGF e encontraram um total de 23 artigos publicados em periódicos científicos desde 2001. O levantamento também localizou 30 trabalhos apresentados nas primeiras quatro edições do congresso anual do Instituto Brasileiro de Avaliação Psicológica. Apesar de a produção nacional ser ainda pequena quando comparada ao volume das publicações internacionais (vide as meta-análises de Digman, 1997; Rushton \& Irwing, 2008; van der Linden \& et al., 2010; Zawadzki \& Strelau, 2010; e o estudo transcultural de Hendriks \& et al, 2003), as autoras apontaram para um padrão de crescimento no número de publicações (Silva \& Nakano, 2011). Isso mostra que o modelo dos CGF tem desempenhado um papel importante nas investigações sobre tópicos relacionados à personalidade no contexto brasileiro.

Atualmente, existem diversos inventários de auto relato para a avaliação da personalidade no modelo dos CGF desenvolvidos ou adaptados e que estão disponíveis no Brasil. Exemplos são: NEO-Personality Inventory-Revised (NEO-PI-R; Costa \& McCrae, 2007), Big Five 
Inventory (BFI; Andrade, 2008; Benet-Martínez \& John, 1998) e Bateria Fatorial da Personalidade (BFP). Existem também instrumentos desenvolvidos para a avaliação de dimensões específicas (e de suas respectivas facetas), como a Escala Fatorial de Extroversão (EFEx; Nunes \& Hutz, 2007a), a Escala Fatorial de Ajustamento Emocional/Neuroticismo (EFN; Hutz \& Nunes, 2001), a Escala Fatorial de Socialização (EFS; Nunes \& Hutz, 2007b) e a Escala de Abertura a Experiências (EAE; Vasconcellos \& Hutz, 2008). Esses instrumentos são bastante adequados para o uso clínico e para muitas situações de pesquisa, uma vez que proporcionam uma avaliação compreensiva da personalidade. Alguns desses, como o NEO-PI-R, a BFP e a EFEx, avaliam também as diversas facetas de cada dimensão da personalidade. Todavia, em muitas situações práticas, instrumentos compreensivos com grande número de itens podem representar um desafio metodológico, em função do tempo que demandam para resposta por parte do indivíduo avaliado. Dessa maneira, por exemplo, pode ser vantajoso utilizar medidas breves em ocasiões em que são necessárias avaliações rápidas ou procedimentos de triagem. Outras situações são estudos amplos com a aplicação de diversos instrumentos em pouco tempo e estudos que requerem que os participantes respondam a instrumentos a respeito de si mesmos e também sobre outros indivíduos (Mullins-Sweatt, Jamerson, Samuel, Olson, \& Widiger, 2006).

\section{Medidas breves para a avaliação dos CGF}

Tendo em vista sua utilidade em diversos contextos de pesquisa, cada vez mais os autores têm investigado evidências de validade de medidas breves para a avaliação da personalidade no modelo dos CGF (Gosling, Rentfrow, \& Swann, 2003; Herzberg \& Brähler, 2006; Mlačić, \& Goldberg, 2007; Muck, Hell, \& Gosling, 2007; Saucier, 1994; Thompson, 2008). As principais medidas breves para avaliar os CGF são compostas por adjetivos corriqueiramente empregados pelos indivíduos leigos para descrever características da personalidade, denominados "marcadores". Medidas baseadas em marcadores têm sido utilizadas desde a década de 60 do século passado (e.g., Norman \& Goldberg, 1966), sendo o trabalho de Saucier (1994) uma das primeiras tentativas de criar um instrumento breve, reduzindo os 100 marcadores de Goldberg (1992) para apenas 40. Marcadores da personalidade são capazes de reduzir ainda mais o tempo da aplicação quando comparados a inventários breves constituídos por itens-sentença, sendo o seu uso frequente na pesquisa sobre a personalidade em diversos contextos culturais (e.g., García, Aluja, \& García, 2004; Goldberg, 1992; Herzberg \& Brähler, 2006; Mlačić, \& Goldberg, 2007; Muck et al., 2007; Saucier, 1994; Thompson, 2008).

No Brasil, Hutz et al. (1998) desenvolveram marcadores da personalidade com base no trabalho de Goldberg (1992). O estudo contou com uma amostra de 976 estudantes universitários e utilizou análises fatoriais com diversos métodos de extração e rotação. Os resultados permitiram estabelecer uma solução final de cinco fatores com 64 adjetivos marcadores. Todavia, na literatura internacional, instrumentos breves com marcadores apresentam tipicamente um número bem menor de marcadores (e.g., 16 adjetivos no estudo de Herzberg \& Brähler, 2006). Dessa forma, o objetivo do presente estudo foi elaborar uma versão reduzida dos marcadores desenvolvidos por Hutz et al. (1998), avaliando suas propriedades psicométricas em uma amostra de estudantes universitários. A seleção dos itens foi baseada em uma combinação de critérios teórico-semânticos e estatísticos e buscou maximizar a validade de conteúdo das subescalas, apesar do reduzido número de marcadores.

\section{Método}

\section{Participantes}

A amostra foi constituída por 674 estudantes universitários de duas universidades do Estado do Rio Grande do Sul, uma federal e outra particular. A amostra foi definida por conveniência. A média de idades foi de 23,48 anos $(D P=$ $6,46)$, sendo $54,6 \%$ dos indivíduos do sexo feminino.

\section{Instrumento}

Foram utilizados os adjetivos marcadores para a avaliação da personalidade no modelo dos CGF desenvolvidos por Hutz e et al. (1998). Esse instrumento é composto por 64 adjetivos divididos em cinco subescalas, que complementam o enunciado "Eu sou uma pessoa...". Foi utilizada uma escala Likert de cinco pontos, sendo $1=$ Discordo totalmente e $5=$ Concordo totalmente. Os autores desse instrumento reportaram Alphas de Cronbach adequados para as subescalas do instrumento, variando de 0,78 a 0,88 .

\section{Procedimentos}

O projeto de pesquisa foi aprovado pelo Comitê de Ética em Pesquisa do Instituto de Psicologia da Universidade Federal do Rio Grande do Sul. Foram obtidos termos de consentimento dos participantes antes de estes responderem aos instrumentos de pesquisa. Todas as coletas foram feitas em sala de aula, de forma coletiva e seguindo procedimentos padronizados, fazendo parte de um estudo maior sobre motivos para usar bebidas alcoólicas. Foram registrados os adjetivos que geraram dificuldade de compreensão pelos participantes.

\section{Análise dos dados}

Foram realizadas análises fatoriais exploratórias com método de extração Maximum Likelihood e rotação oblíqua Direct Oblimin, com o software SPSS 17.0. A escolha dessa técnica de análise se deu por dois motivos: 1) o intuito de analisar apenas a variância comum entre os itens, removendo a variância única de cada item; e 2) a necessidade de considerar a possibilidade de correlações entre os fatores do instrumento, corriqueiramente relatadas nos estudos na área (e.g., Rushton \& Irwing, 2008). Para a retenção dos fatores, 
foram executadas análises paralelas com o software Monte Carlo PCA, com autovalores gerados a partir de 500 matrizes de correlação com dados aleatórios. O critério de comparação foi definido como sendo o percentil 95 da distribuição dos autovalores aleatórios. O objetivo da aplicação da análise paralela foi reter apenas fatores com variância explicada maior do que aquela esperada ao acaso (Horn, 1965).

\section{Resultados}

Uma análise exploratória dos dados revelou que todos os marcadores apresentarem violações significativas $(p<$ $0,05)$ da normalidade, de acordo com o teste de Kolmogorov-Smirnov. Todavia, uma inspeção também mostrou que nenhum dos 64 marcadores iniciais apresentou assimetria ou curtose com valores acima de $|2,00|$ (apenas sete dos 64 marcadores apresentaram assimetria ou curtose $>|1,0|$ ). Assim, considerando-se que o índice Kaiser-Meyer-Olkin foi de 0,82 e o teste de esfericidade de Bartlett apresentou $p<0,001$, os dados foram considerados adequados para as análises fatoriais, apesar dos pequenos desvios da normalidade. Os autores então procederam a uma combinação de critérios teórico-semânticos e estatísticos para a escolha dos marcadores para a versão reduzida.

Em primeiro lugar, foi executada uma análise fatorial com todos os 64 marcadores, forçando-se uma solução de cinco fatores para situar os marcadores nas suas referidas dimensões. O passo seguinte foi eleger marcadores que contemplassem, quando possível, o conteúdo das diferentes facetas dos CGF como descritas em Paunonen e Ashton (2001). Assim, buscou-se manter marcadores que representassem o conteúdo das facetas dos CGF, apesar do fato de o instrumento original não ter sido elaborado com o propósito de necessariamente avaliá-las. Procurou-se evitar manter marcadores com mesmo conteúdo semântico. Ao mesmo tempo, foram eliminados marcadores com carga fatorial abaixo de 0,30 e também aqueles apontados como de difícil compreensão pelos participantes (e.g., "afável", "metódica", "assídua"). Após, buscou-se manter um mesmo número de itens por fator, uma vez que a diferença no número de itens poderia resultar em diferença também na consistência interna das subescalas. Foram estabelecidos cinco marcadores para cada dimensão, em um total de 25.

A seguir, uma nova análise fatorial foi executada, com o objetivo de avaliar a estrutura final dos 25 marcadores escolhidos. Foram extraídos seis fatores com autovalor $>$ 1, explicando $58,54 \%$ da variância comum entre dos itens. Esses autovalores foram então comparados com aqueles obtidos pela análise paralela. Observou-se que, dentre os autovalores observados a partir da análise fatorial, apenas os cinco primeiros excederam em explicação os autovalores aleatórios $(4,50$ versus $1,37,3,55$ versus $1,31,2,20$ versus $1,27,1,72$ versus 1,23 e 1,51 versus 1,20 ). Também o gráfico de screeplot permitiu uma interpretação de cinco fatores. Dessa forma, foi mantida uma solução de cinco fatores explicando $53,92 \%$ da variância comum dos itens, sendo os marcadores e as respectivas cargas apresentadas na Tabela 1. Médias, desvios-padrão e correlações entre as mesmas podem ser visualizadas na Tabela 2 .
Tabela 1. Cargas Fatoriais dos Marcadores da Personalidade e Alphas de Cronbach para as Subescalas.

\begin{tabular}{|c|c|c|c|c|c|}
\hline & $\mathrm{E}$ & S & $\mathrm{C}$ & $\mathrm{N}$ & A \\
\hline Comunicativa & 0,79 & & & & \\
\hline Quieta & $-0,74$ & & & & \\
\hline Tímida & $-0,71$ & & & & \\
\hline Desembaraçada & 0,65 & & & & \\
\hline Inibida & $-0,50$ & & & & \\
\hline Amável & & 0,68 & & & \\
\hline Gentil & & 0,62 & & & \\
\hline Simpática & & 0,61 & & & \\
\hline Bondosa & & 0,60 & & & \\
\hline Compreensiva & & 0,59 & & & \\
\hline Dedicada & & & $-0,81$ & & \\
\hline Esforçada & & & $-0,75$ & & \\
\hline Responsável & & & $-0,64$ & & \\
\hline Organizada & & & $-0,57$ & & \\
\hline Cuidadosa & & & $-0,42$ & & \\
\hline Pessimista & & & & 0,61 & \\
\hline Deprimida & & & & 0,54 & \\
\hline Insegura & & & & 0,54 & \\
\hline Ansiosa & & & & 0,52 & \\
\hline Aborrecida & & & & 0,47 & \\
\hline Criativa & & & & & 0,65 \\
\hline Artística & & & & & 0,64 \\
\hline Filosófica & & & & & 0,47 \\
\hline Aventureira & & & & & 0,32 \\
\hline Audaciosa & & & & & 0,32 \\
\hline Alphas de Cronbach & 0,83 & 0,79 & 0,79 & 0,69 & 0,61 \\
\hline
\end{tabular}

Nota $. \mathrm{E}=$ Extroversão, $\mathrm{S}=$ Socialização, $\mathrm{C}=$ Conscienciosidade, $\mathrm{N}=$ Neuroticismo, $\mathrm{A}=$ Abertura

É possível observar na Tabela 1 que, com exceção dos marcadores "aventureira" e "audaciosa", todos apresentaram correlações moderadas ou altas com seus respectivos fatores. Os Alphas de Cronbach para as subescalas formadas a partir da média dos itens em cada fator ficaram dentro do intervalo de valores de 0,61 a 0,83, indicando um nível de razoável a satisfatório para a consistência interna das subescalas. As médias das subescalas, a seu turno, com exceção de neuroticismo, ficaram todas acima do ponto médio $(3,00)$ da escala Likert empregada, como visto na Tabela 2. Assim, a maioria das pessoas atribuiu valores altos na escala para marcadores característicos de bom ajuste social (extroversão, socialização, conscienciosidade e abertura) e valores baixos na escala para marcadores típicos de desajuste (neuroticismo). 
Tabela 2. Médias, Desvios-padrão e Correlações das Subescalas.

\begin{tabular}{|c|c|c|c|c|c|c|c|}
\hline Subescala & 1 & 2 & 3 & 4 & 5 & $M$ & $D P$ \\
\hline 1. Extroversão & - & $0,12^{*}$ & 0,06 & $-0,33 * *$ & $0,19 * *$ & 3,36 & 0,90 \\
\hline 2. Socialização & & - & $0,40 * *$ & $-0,04$ & $0,30 * *$ & 3,89 & 0,66 \\
\hline 3. Conscienciosidade & & & - & $-0,11 *$ & $0,15^{* *}$ & 3,88 & 0,74 \\
\hline 4. Neuroticismo & & & & - & 0,01 & 2,39 & 0,73 \\
\hline 5. Abertura & & & & & - & 3,13 & 0,73 \\
\hline
\end{tabular}

Nota. $M=$ média, $D P=$ desvio-padrão. $* p<0,01, * * p<0,001$.

\section{Discussão}

O objetivo do presente estudo foi elaborar uma versão reduzida dos marcadores para a avaliação da personalidade no modelo dos CGF desenvolvidos por Hutz e at al. (1998). A seleção dos marcadores para a composição do instrumento reduzido se deu através de uma combinação de critérios teórico-semânticos e estatísticos. Buscou-se manter marcadores de fácil compreensão, sendo proposta uma medida reduzida composta por apenas 25 marcadores distribuídos em cinco fatores que corresponderam às expectativas teóricas.

A consistência interna para as subescalas (entre $0,61 \mathrm{e}$ 0,83 ) resultantes foi comparável à de outros instrumentos reduzidos relatados na literatura (Herzberg \& Brähler, 2006; Mlačić, \& Goldberg, 2007; Muck, Hell, \& Gosling, 2007). Teria sido possível aumentar a consistência interna das subescalas, selecionando marcadores a partir do pool inicial (de 64 marcadores) unicamente com base nas melhores cargas fatoriais. Contudo, esse procedimento teria possíveis repercussões negativas na validade de conteúdo do instrumento, uma vez que maximizaria a redundância entre os marcadores mantidos. Assim, é importante mencionar que um estudo nacional recente mostrou que é possível a redução de uma medida dos CGF sem que haja perda de informação em termos da amplitude do traço latente avaliada (Nunes, Muniz, Nunes, Primi, \& Miguel, 2010). Entretanto, serão necessários novos estudos com Teoria de Resposta ao Item a fim de avaliar aspectos como as funções de informação de cada escala e se os itens apresentam um nível de dificuldade variável, representativo de diferentes aspectos de cada dimensão dos CGF.

Outro aspecto digno de nota foi a existência de correlações significativas entre as subescalas do instrumento. Ainda que extroversão e abertura tenham apresentado $r=0,19$, de maneira inconsistente com a literatura sobre os fatores Alpha e Beta (em que extroversão e abertura apresentam correlações moderadas, como em Digman, 1997; e Rushton \& Irwing, 2008), não se descarta a possibilidade de que um modelo hierárquico possa representar satisfatoriamente a estrutura do instrumento. Como revisado anteriormente, uma perspectiva oblíqua pode ter como implicação teórica a existência de uma hierarquia de fatores superiores aos CGF, sendo possível não apenas os fatores Alpha, Beta, mas também um FGP (Musek, 2007; Rushton \& Irwing, 2008; van der Linden et al., 2010). É recomendável, assim, que estudos posteriores com amostras ampliadas sejam realizados para investigar a plausibilidade de estruturas fatoriais complexas para os marcadores reduzidos através de técnicas confirmatórias. Ressalta-se também a necessidade de investigações sobre a validade convergente com outros instrumentos nacionais de avaliação dos CGF, como a BFP (Nunes \& et al., 2010) e a EAE (Vasconcellos \& Hutz, 2008). As correlações com critérios externos também devem ser estimadas, comparando-se com resultados obtidos com os instrumentos compreensivos tradicionais.

Os marcadores reduzidos propostos pelo presente estudo podem cumprir um papel importante em diversas situações práticas ou em estudos cujo delineamento impossibilita a utilização de instrumentos com maior número de itens. Segundo Gosling et al. (2003), há circunstâncias em que o pesquisador se vê frente a um dilema e deve decidir entre usar uma medida breve para avaliar a personalidade ou então não usar nenhuma medida desse tipo. Dessa maneira, o presente instrumento não se destina a substituir em definitivo o uso de instrumentos compreensivos, mas sim fornecer aos pesquisadores brasileiros economia de tempo e de recursos em condições específicas de avaliação. Em virtude disso, considera-se o presente instrumento promissor para a pesquisa na área da avaliação da personalidade no Brasil.

O presente estudo buscou suprir uma lacuna na literatura nacional e apresentar uma medida breve dos CGF que pode ser de utilidade em diversas áreas de estudo e de exercício profissional dentro da Psicologia. Uma importante limitação metodológica deste trabalho foi o fato de que a amostra utilizada não foi obtida de forma randomizada, de modo que se faz necessário novos estudos de evidências de validade com amostras ampliadas. O teste confirmatório da estrutura do instrumento em delineamentos multigrupos pode ser de grande valia para a estimação da estabilidade fatorial da medida em estudos futuros. Ainda assim, os resultados aqui apresentados são bastante promissores e sugerem a adequação psicométrica do instrumento.

\section{Referências}

Andrade, J. M. (2008). Evidências de validade do Inventário dos Cinco Grandes Fatores de personalidade para o Brasil. Tese de Doutorado, Universidade de Brasília, Brasília, DF.

Bäckström, M., Björklund, F., \& Larsson, M. R. (2009). Fivefactor inventories have a major general factor related to social desirability which can be reduced by framing items neutrally. Journal of Research in Personality, 43, 335-344. 
Bartholomeu, D., Nunes, C. H. S. S., \& Machado, A. A. (2008). Traços de personalidade e habilidades sociais em universitários. Psico-USF, 13(1), 41-50.

Benet-Martínez, V., \& John, O. P. (1998). "Los Cinco Grandes" Across cultures and ethnic groups: Multitrait-multimethod analyses of the Big Five in Spanish and English. Journal of Personality and Social Psychology, 75, 729-750.

Bouchard, T. J., \& Loehlin, J. C. (2001). Genes, evolution, and personality. Behavior Genetics, 31(3), 243-273.

Costa, P. T., Jr., \& McCrae, R. R. (2007). NEO PI-R: Inventário de personalidade $N E O$ revisado e inventário de cinco fatores NEO revisado NEO-FFI-R [Versão curta]. São Paulo: Vetor Editora Psico-Pedagócica.

Digman, J. M. (1990). Personality structure: Emergence of the five-factor model. Annual Review of Psychology, 41, 417-440.

Digman, J. M. (1997). Higher-order factors of the Big Five. Journal of Personality and Social Psychology, 73(6), 1246-1256.

García, O., Aluja, A., \& García, L. F. (2004). Psychometric properties of Goldberg's 50 personality markers for the Big Five model. European Journal of Psychological Assessment, 20(4), 310-319.

Goldberg, L. R. (1992). The development of markers for the BigFive factor structure. Psychological Assessment, 4, 26-42.

Goldberg, L. R. (1993). The structure of phenotypic personality traits. American Psychologist, 48(1), 26-34.

Gosling, S. D., Rentfrow, P. J., \& Swann, W. B., Jr. (2003). A very brief measure of the Big-Five personality domains. Journal of Research in Personality, 37, 504-528.

Hendriks, A. A., Perugini, M., Angleitner, A., Ostendorf, F., Johnson, J. A., de Fruyt, F., Hřebíčková, M., Kreitler, S., Murakami, T., Bratko, D., Conner, M., Nagy, J., Rodríguez-Fornells, A., \& Ruisel, I. (2003). Five-Factor Personality Inventory: Crosscultural generalizability across 13 countries. European Journal of Personality, 17, 347-373.

Herzberg, P. Y., \& Brähler, E. (2006). Assessing the big-five domains via short forms: A cautionary note and a proposal. European Journal of Psychological Assessment, 22(3), 139-148.

Horn, J. L. (1965), A rationale and test for the number of factors in factor analysis. Psychometrika, 30, 179-85.

Hutz, C. S., \& Nunes, C. H. S. S. (2001). Escala Fatorial de Extroversão - Manual técnico. São Paulo: Casa do Psicólogo.

Hutz, C. S., Nunes, C. H. S. S., Silveira, A. D., Serra, J., Anton, M., \& Wieczorek, L. S. (1998). The development of the big five markers for personality assessment in Brazil. Psicologia: Reflexão e Crítica, 11, 395-411.

John, O. P., Angleitner, A., \& Ostendorf, F. (1988). The lexical approach to personality: A historical review of trait taxonomic research. European Journal of Personality, 2, 171-203.

Just, C. (2011). A review of the literature on the general factor of personality. Personality and Individual Differences, 50, $765-771$.
Kandler, C., Bleidorn, W., Riemann, R., Spinath, F. M., Thiel, W., $\&$ Angleitner, A. (2010). Sources of cumulative continuity in personality: A longitudinal multiple-rater twin study. Journal of Personality and Social Psychology, 98(6), 9951008.

Mlačić, B., \& Goldberg, L. R. (2007). An analysis of a cross-cultural personality inventory: The IPIP Big-Five Factor Markers in Croatia. Journal of Personality Assessment, 88(2), 168-177.

Mount, M. K., Barrick, M. R., Scullen, S. M., \& Rounds, J. (2005). Higher-order dimensions of the big five personality traits and the big six vocational interest types. Personnel Psychology, $58,447-478$.

Muck, P. M., Hell, B., \& Gosling, S. D. (2007). Construct validation of a short five-factor model instrument: A self-peer study on the German adaptation of the Ten-Item Personality Inventory (TIPI-G). European Journal of Psychological Assessment, 23(3), 166-175.

Mullins-Sweatt, S. N., Jamerson, J. E., Samuel, D. B., Olson, D. R., \& Widiger, T. A. (2006). Psychometric properties of an abbreviated instrument of the Five-Factor Model. Assessment, 13(2), 119-137.

Musek, J. (2007). A general factor of personality: Evidence for the Big One in the five-factor model. Journal of Research in Personality, 41, 1213-1233.

Norman, W. X., \& Goldberg, L. R. (1966). Raters, ratees, and randomness in personality structure. Journal of Personality and Social Psychology, 4, 681-691.

Nunes, C. H. S. S. (2007). Lançamento da Escala Fatorial de Extroversão (EFEx) e Escala Fatorial de Socialização. Avaliação Psicológica, 6(1), 103-106.

Nunes, C. H. S. S., \& Hutz, C. S. (2007a). Escala Fatorial de Extroversão-Manual técnico. São Paulo: Casa do Psicólogo.

Nunes, C. H. S. S., \& Hutz, C. S. (2007b). Escala Fatorial de Socialização-Manual técnico. São Paulo: Casa do Psicólogo.

Nunes, C. H. S. S., Hutz, C. S., \& Giacomoni, C. H. (2009). Associação entre bem-estar subjetivo e personalidade no modelo dos Cinco Grandes Fatores. Avaliação Psicológica, 8(1), 99-108.

Nunes, M. F. O., Muniz, M., Nunes, C. H. S. S., Primi, R., \& Miguel, F. K. (2010). Escala de Socialização - versão reduzida: seleção de itens e propriedades psicométricas. Psicologia: Reflexão e Crítica, 23(2), 345-353.

Ozer, D., \& Benet-Martínez, V. (2006). Personality and the prediction of consequential outcomes. Annual Review of Psychology, 57, 401-421.

Paunonen, S. V., \& Ashton, M. C. (2001). Big Five factors and facets and the prediction of behavior. Journal of Personality and Social Psychology, 81(3), 524-539.

Revelle, W. (1995). Personality processes. Annual Review of Psychology, 46, 295-328.

Ruiz, V. M., \& Jiménez, J. A. (2004). Estructura de la personalidade: Ortogonalidad versus oblicuidad. Anales de Psicología, 20(1), $1-13$. 
Rushton, J. P., \& Irwing, P. (2008). A General Factor of Personality (GFP) from two meta-analyses of the Big Five: Digman (1997) and Mount, Barrick, Scullen, and Rounds (2005). Personality and Individual Differences, 45, 679-683.

Saucier, G. (1994). Mini-Markers: A brief version of Goldberg's unipolar Big-Five markers. Journal of Personality Assessment, 63, 506-516.

Silva, I. B., \& Nakano, T. C. (2011). Modelo dos Cinco Grandes Fatores da personalidade: análise de pesquisas. Avaliação Psicológica, 10(1), 51-62.

Sisto, F. F., \& Oliveira, A. F. (2007). Traços de personalidade e agressividade: um estudo de evidência de validade. Psic, 8(1), 89-99.

Thompson, E. R. (2008). Development and validation of an International English Big-Five Mini-Markers. Personality and Individual Differences, 45, 542-548.

Van der Linden, D., Te Nijenhuis, J., \& Bakker, A. B. (2010). The General Factor of Personality: A meta-analysis of big five intercorrelations and a criterion-related validity study. Journal of Research in Personality, 44(3), 315-327.
Vasconcellos, S. J. L., \& Hutz, C. S. (2008). Construção e validação de uma escala de abertura à experiência. Avaliação Psicológica, 7(2), 135-141.

Zawadzki, B., \& Strelau, J. (2010). Structure of personality: Search for a general factor viewed from a temperament perspective. Personality and Individual Differences, 49, 77-82.

Recebido em 19.11.2010

Primeira decisão editorial em 19.01.2012

Versão final em 05.03.2012

Aceito em 25.04.2012 